Published Online on 29 June 2016

Proc Indian Natn Sci Acad 82 No. 2 June Spl Issue 2016 pp. 163-172

DOI: $10.16943 /$ ptinsa/2016/48410

Research Paper

\title{
Robust Finite Element Approaches to Systemic Circulation Using the Locally Conservative Galerkin (LCG) Method
}

\author{
HAYDER M HASAN ${ }^{1, \#}$ and PERUMAL NITHIARASU ${ }^{1}$, * \\ ${ }^{1}$ Biomedical Engineering and Rheology Group, Zienkiewicz Centre for Computational Engineering, \\ Swansea University, Swansea SA2 8PP, United-Kingdom
}

(Received on 03 February 2016; Accepted on 25 February 2016)

\begin{abstract}
In this paper novel finite element algorithms for robustly solving flow equations of a systemic circulation are presented and compared. A novel, Locally Conservative Galerkin (LCG) method is developed by adopting semi- and fully- implicit time discretisations to address the behavior of blood flow in the human circulatory system. These techniques are efficient for nonlinear system of equations used in blood flow models and achieve rapid convergence. Several comparisons are made between the methods to demonstrate the validity and stability of the current numerical models to solve the blood flow characteristics in a human body.
\end{abstract}

Keywords: Finite Element Method; Explicit, Semi-and Fully-Implicit Locally Conservative Galerkin (LCG) Methods; Systemic Circulation; Blood Flow

\section{Introduction}

One-dimensional blood flow simulation has proven to be an efficient tool to describe the flow characteristics in a human circulatory system. Since the width of the pressure wave produced by the heart is much greater than any vessel diameter, the one-dimensional assumption is valid for studying systemic circulation (Avolio, 1980; Alastruey et al., 2007; Steele et al., 2007; Mynard and Nithiarasu, 2008; Raines et al., 1974; Wan et al., 2002; Low et al., 2012; Stergiopulos et al., 1992; Chen et al., 2013; Blanco et al., 2012; Kufahl and Clark, 1985; Urquiza et al., 2006; Franke et al., 2002; Watanabe et al., 2013). Some of the recent publications in this area adopted Locally Conservative Galerkin (LCG) technique, which was developed in (Nithiarasu, 2004, Thomas et al., 2008) using an explicit Taylor-Galerkin method along with the characteristic variables. The characteristic variables in LCG method are employed to prescribe boundary and other conditions at various locations of an arterial tree. Often, the characteristic variable information for the boundary conditions are obtained from the previous time step. Although such method is more intuitive due to the wave nature being exploited, the explicit method appears to lead to stability issues (Malossi et al., 2012), especially at branching points. One of the remedies recommended is a two time step method in which the first time step corresponds to the stability limit of an explicit configuration of each segment (local time step), and the global time step is for the branching sites (Malossi et al., 2012). A similar technique was also used by (Blanco et al., 2011) to overcome difficulties of using two different time steps, and to avoid previous time step information. As a result of the implicit treatment in the reported works, the time step restriction associated with the explicit treatment was reduced. However in such methods, the boundary conditions and treatment of branches must be carried out in advance, which may lead to additional restrictions and less robust solution procedure.

*Author for Correspondence: E-mail: P.Nithiarasu@swansea.ac.uk

${ }^{\#}$ On study leave from Shatra Technical Institute, Southern Technical University, Ministry of Higher Education and Scientiûc Research, Iraq 
While explicit strategy is computationally robust, simple, memory efficient and often more accurate, the main drawback of such methods is the time step restriction. On the other hand, the main advantage of the implicit method is that no limitation on the time step used but the method is difficult to implement. The iterative solution of a non-linear and nonsymmetric matrix may not converge for all parameters when implicit method is employed. Such an observation is demonstrated by (Wanga et al. 2015) via omparisons of the performance of four numerical schemes, namely MacCormack, Taylor Galerkin, monotonic upwind scheme for conservation law and local discontinuous Galerkin method. Among the four methods, Taylor-Galerkin method was found to be the fastest with a comparable accuracy, but it becomes slower if a large number of elements is used. Nonetheless, such restrictions can be avoided with an LCG method (Nithiarasu 2004, Thomas et al., 2008) in which storage and inversion of matrices are limited to element matrices. To reduce the memory limitation of continuous Galerkin method and to reduce the explicit time step restrictions, we propose some novel techniques to obtain better stability, speed and robustness. The methods proposed iteratively solves the matrices element-by-element for the entire physical domain of the circulatory system. Moreover, various versions of LCG method are compared against each other to demonstrate the relative accuracy.

\section{Outline of the Physical Model}

The physical model consists of a network of elastic tubes representing blood flow in large arteries, i.e., 63 arteries including the coronaries (Mynard and Nithiarasu, 2008; Low et al., 2012; Coccarelli and Nithiarasu, 2015; Boileau et al., 2015; Coccarelli and Nithiarasu, 2015; Coccarelli et al., 2016). To represent the arterial system, the model proposed by (Mynard and Nithiarasu, 2008; Low et al., 2012) is adopted and for 63 arterial segments are used as shown in Fig. 1. This is a branching network characterised by bifurcations and cross sectional discontinuities. The reflections due to network singularities and terminals are also incorporated into the model. The fluid system is described by two variables: the cross sectional area $A$ and the average values of velocity $u$. The flow is considered incompressible, laminar and pressure $p$ is linked to the cross sectional area via a nonlinear relationship proposed by (Formaggia et al., 2002;

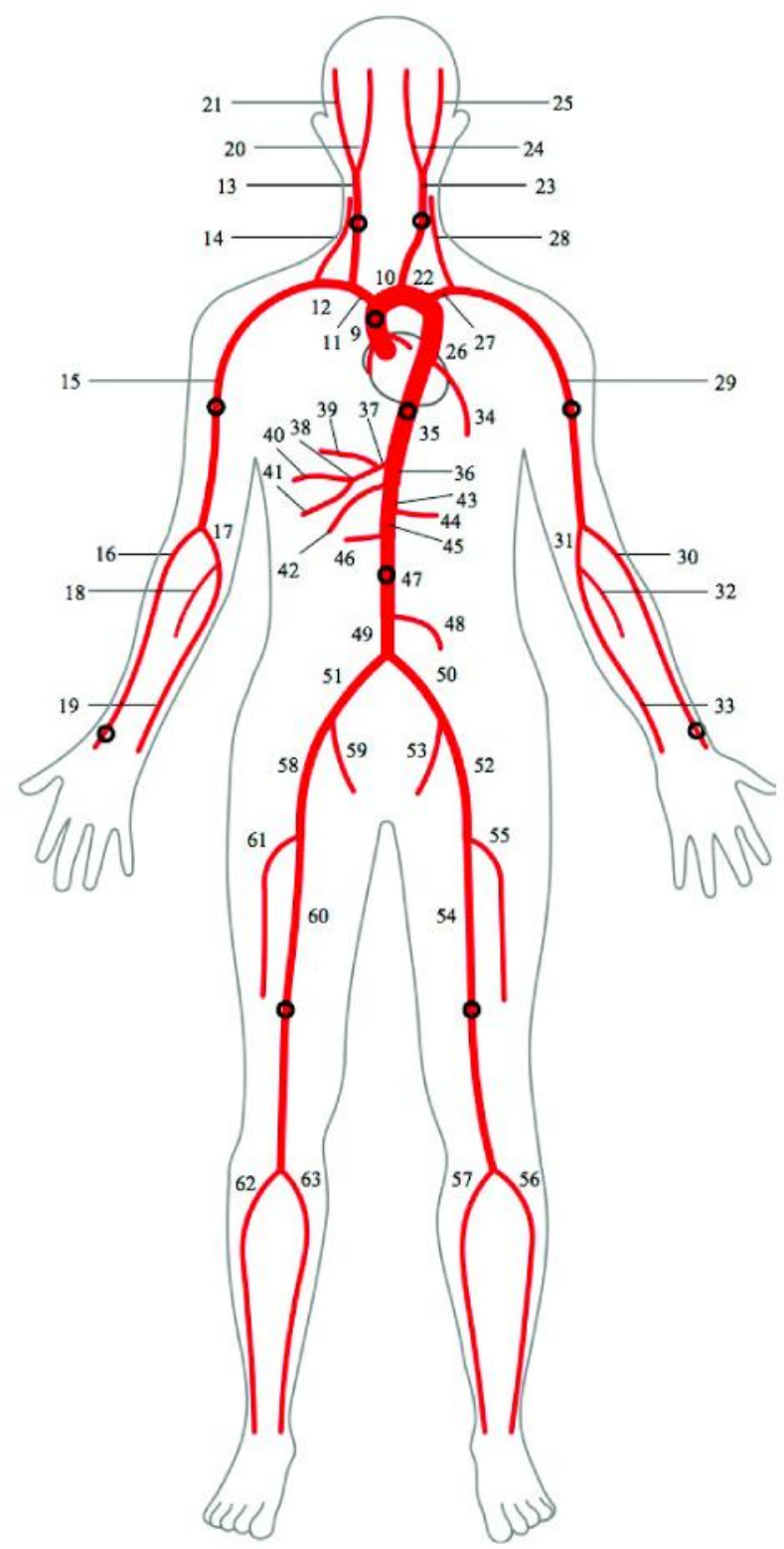

Fig. 1: Configuration of the arterial tree for the current module Low et al. (2012)

Olufsen et al., 2000) which can be written as

$$
p=p_{\text {ext }}+\beta\left(\sqrt{A}-\sqrt{A}_{0}\right)
$$

where $p_{\text {ext }}$ is the pressure from the surrounding tissues, $A$ is the area of cross section, $A_{o}$ is the area at zero transmural pressure and $\beta$ accounts for the material properties of the elastic vessel and given by

$$
\beta=\frac{\sqrt{\pi} h E}{A_{0}(1-\sigma)}
$$


where $h$ is the vessel wall thickness, $E$ is Young's modulus and $\sigma$ is the Poisson's ratio, assumed to be 0.5 (i.e. the vessel wall is incompressible). The conservation of mass and momentum equations may be written as (Mynard and Nithiarasu, 2008; Sherwin et al., 2003),

$$
\frac{\partial A}{\partial t}+\frac{\partial(A u)}{x}=0
$$

and

$$
\frac{\partial u}{\partial t}+u \frac{\partial u}{\partial x}+\frac{1}{\rho} \frac{\partial p}{\partial x}-\frac{1}{\rho} \frac{\partial \tau}{\partial x}=0
$$

In the above equation $\rho$ is the density of the blood and the viscous term may be rewritten using Poiseuille flow assumption. In such a case, the flow is assumed to be fully developed and steady and can be represented as

$$
\frac{\partial p}{\partial t}=\frac{\partial \tau}{\partial x}=-\frac{8 \pi \mu u}{A}
$$

The conservation laws for flow in large arteries can be written in terms of characteristic variables. They are useful for prescribing inlet and outlet boundary conditions and also for transmitting information in discontinuities between segments (Mynard and Nithiarasu, 2008). At the fluid inlet point, the aortic valve is modelled, while the pumping action of the heart is modelled as a prescribed forward pressure source (Mynard and Nithiarasu, 2008; Low et al., 2012). To model branch ending, tapering vessels are used. In the present work, three different algorithms are used to solve the flow equations.

\section{Proposed Finite Element Flow Algorithms}

An iterative strategy, namely Newton-LU decomposition (factorization), is adopted here. The difference from other iterative methods is that we use element-by-element solution, which results in an inexpensive and very fast convergence. The main goal is to achieve the fast convergence with an acceptable accuracy and stability, especially in complex problems such as human arterial system in which many segments and branches create a complex network of passages. Among all methods, Newton procedure is still a very efficient tool to solve a set of non-linear equations, and also LU decomposition for solving the matrix. Since we divide the domain into many subdomains (i.e., elements), the LCG method needs only to deal with very small matrix size of $4 \times 4$ per element, and therefore almost all solvers are applicable with manageable Jacobian matrix. Since the size of the matrix is unaffected by the total number of nodes, the standard problems of convergence and additional coupling between nodes are reduced here. This is one of the main advantages of LCG technique, which produces a consistent matrix regardless of the number of elements in the domain. Obviously, this allows us to achieve the solution to any number of arteries.

By substituting Eq. 1 into Eq. 4 results in the closed system of the primitive variables, which can be written in a compact form as (Mynard and Nithiarasu 2008).

$$
\frac{\partial \mathbf{U}}{\partial t}=\frac{\partial \mathbf{F}}{\partial x}-\mathbf{S}=0
$$

where

$$
\mathbf{U}=\left[\begin{array}{l}
A \\
u
\end{array}\right], \mathbf{F}=\left[\begin{array}{c}
A u \\
\frac{u^{2}}{2}+\frac{1}{\rho}\left[p_{\text {ext }}+\beta\left(\sqrt{A}-\sqrt{A_{0}}\right)\right]
\end{array}\right]
$$

and

$$
\mathbf{S}=\left[\begin{array}{c}
0 \\
-\frac{8 \pi \mu}{\rho} \frac{u}{A}
\end{array}\right]
$$

Applying the Galerkin method to Eq. 6 may produce instability due to the presence of the first order derivative of $F$. Thus, upwinding type approach is often needed. For example, Taylor expansion based methods have been used to avoid instability (Mynard and Nithiarasu, 2008; Low et al., 2012; Coccarelli and Nithiarasu, 2015; Coccarelli et al., 2016). By applying such method, the semi descrete form of Eq. 6 can be written as (Mynard and Nithiarasu, 2008)

$$
\begin{aligned}
& \frac{\mathbf{S}^{n+1}+\mathbf{U}^{n}}{\Delta t}=\mathbf{S}^{n+\theta}-\frac{\partial \mathbf{F}^{n+\theta}}{\partial x}-\frac{\Delta t}{2} \\
& {\left[\frac{\partial}{\partial x}\left(\mathbf{B S}-\mathbf{B} \frac{\partial \mathbf{F}}{\partial x}\right)-\mathbf{C} \frac{\partial \mathbf{F}}{\partial x}-\mathbf{C S}\right]^{n+\theta}}
\end{aligned}
$$


where

$\mathbf{B}$ and $\mathbf{C}$ are two Jacobian matrices, given as

$$
\mathbf{B}=\frac{\partial \mathbf{F}}{\partial \mathbf{U}} \quad \text { and } \quad \mathbf{C}=\frac{\partial \mathbf{S}}{\partial \mathbf{U}}
$$

In Eq. 7, $\theta=0$ results in fully explicit form and if $\theta=1$ a fully implicit form of the Taylor-Galerkin method is obtained. When $\theta=1$, we adopt the iterative matrix solver.

Applying the fundamentals of finite element method, i.e., linear weighting and shape functions, (readers are referred to (Nithiarasu et al., 2016) for more details) to Eq. 7 gives the fully discrete form of the implicit Global Taylor-Galerkin (GTC) method as $(\theta=1)$,

$$
\begin{aligned}
{[\mathbf{M}]\left\{\Delta \mathbf{U}^{n}\right\}=} & \Delta t\left[[\mathbf{K}]\left\{\mathbf{F}^{n+1}\right\}\right] \\
& +[\mathbf{L}]\left\{\mathbf{S}^{n+1}\right\}+\left\{\mathbf{F}_{\Gamma}\right\}^{n+1}
\end{aligned}
$$

Rewriting Eq. 9 in Locally Conservative TaylorGalerkin (LCTG) form gives

$$
\begin{aligned}
{[\mathbf{M}]_{e}\left\{\Delta \mathbf{U}^{n}\right\}=} & \Delta t\left[[\mathbf{K}]\left\{\mathbf{F}^{n+1}\right\}\right] \\
& \left.+[\mathbf{L}]\left\{\mathbf{S}^{n+1}\right\}+\left\{\mathbf{f}_{\Gamma}\right\}^{n+\theta}\right]_{e}
\end{aligned}
$$

Where subscript $e$ refers to an element. The full details of the matrices $\mathbf{M}, \mathbf{K}$ and $\mathbf{L}$ in Eq. 10 for each element can found in (Mynard and Nithiarasu 2008). The basic methodology of such technique is to treat each element independently, and therefore the physical quantities are enforced by a proper flux between two elements. So, $\mathbf{f}_{\Gamma}$ is used for that purpose (i.e. $\mathbf{f}_{\Gamma}=\mathbf{F}$ ). Such term always goes to zero in the Continuous Taylor Galerkin method except at the boundaries, but because LCG treats each element separately, $\mathbf{f}_{\Gamma}$ represents the element boundaries and therefore, it is kept here. Usually, the method uses an explicit form for the flux, that means $\theta=0$ in Eq. 10 (i.e. $\mathbf{f}_{\Gamma}{ }^{n}=\mathbf{F}^{n}$ ) (Mynard and Nithiarasu, 2008), but here we can use either $\theta=0$ or $\theta=1$ resulting in semi- or fully implicit strategies. Fundamentally, $\mathbf{F}$ depends on the primitive variable of $A$ and $u$ as shown in Eq. 6 . So, if the implicit form is used (i.e. $\theta=1$ ), $\mathbf{f}_{\Gamma}{ }^{n+1}=\mathbf{F}^{n+1}$ is rewritten in terms of implicit area and velocity. At $\theta$ $=0, \mathbf{f}_{\Gamma}{ }^{n}=\mathbf{F}^{n}$ is similarly calculated but at $n$ time level.
In order to solve the above equations, we use Newton method (Zienkiewicz, Taylor and Nithiarasu 2014; Zienkiewicz, Taylor and Fox 2014). Now, let us assume that $\chi$ is an abbreviation of the unknowns and the iterative method takes the form

$$
\chi^{k+1}=\chi^{k}+\delta \chi^{k}
$$

here, $\delta \chi^{k}$ is estimated by Newton iteration, so that

$$
\boldsymbol{J}\left(\chi^{k}\right) \delta \chi^{k}=-R\left(\chi^{k}\right)
$$

Obviously, Eq. 12 requires evaluation of the Jacobian matrix $\boldsymbol{J}$ and the residual $R$ at each iteration. Several methodologies for calculating these quantities have been developed in order to reduce the computational time, which dramatically increases with the number of nodes. However, with current LCTG method there will be no difficulties as the proposed methods results in only $4 \times 4$ matrixes and calculations of these quantities is straightforward. A linear representation of Eq. 12 gives four linear algebraic equations per element (i.e. $R_{A_{1}}, R_{A_{2}}, R_{u_{1}}$ and $R_{u_{2}}$ ). The detailed form of Eq. 12 may now be written as

$$
\left|\begin{array}{l}
\frac{\partial R_{A_{1}}}{\partial_{A_{1}}} \frac{\partial R_{A_{1}}}{\partial_{A_{2}}} \frac{\partial R_{A_{1}}}{\partial_{u_{1}}} \frac{\partial R_{A_{1}}}{\partial_{u_{2}}} \\
\frac{\partial R_{A_{2}}}{\partial_{A_{1}}} \frac{\partial R_{A_{2}}}{\partial_{A_{2}}} \frac{\partial R_{A_{2}}}{\partial_{u_{1}}} \frac{\partial R_{A_{2}}}{\partial_{u_{2}}} \\
\frac{\partial R_{u_{1}}}{\partial_{A_{1}}} \frac{\partial R_{u_{1}}}{\partial_{A_{2}}} \frac{\partial R_{u_{1}}}{\partial_{u_{1}}} \frac{\partial R_{u_{1}}}{\partial_{u_{2}}} \\
\frac{\partial R_{u_{2}}}{\partial_{A_{1}}} \frac{\partial R_{u_{2}}}{\partial_{A_{2}}} \frac{\partial R_{u_{2}}}{\partial_{u_{1}}} \frac{\partial R_{u_{2}}}{\partial_{u_{2}}}
\end{array}\right| \begin{aligned}
& \Delta A_{1} \\
& \Delta A_{2} \\
& \Delta u 1 \\
& \Delta u_{2}
\end{aligned}|=| \begin{gathered}
R A_{1} \\
R A_{2} \\
R u 1 \\
R u 2
\end{gathered} \mid
$$

where subscripts 1 and 2 refer to first and second nodes of a linear element. To obtain the right solution, the set of appropriate boundary conditions must be prescribed at the inlet and the outlet of the domain and here we use the characteristic variables for this purpose. In order to carry out this, we rewrite Eq. 6 in quasi-linear form, i.e.,

$$
\frac{\partial \mathbf{U}}{\partial t}+\mathbf{H} \frac{\partial \mathbf{U}}{\partial x}-\mathbf{S}=0
$$

where $\mathbf{H}$ is Jacobian matrix given by 


$$
\mathbf{H}=\left[\begin{array}{cc}
u & A \\
\frac{\beta}{2 \rho \sqrt{A}} & u
\end{array}\right]
$$

and then the characteristic speeds of the system may be written as (Mynard and Nithiarasu 2008)

$$
\Lambda=\left[\begin{array}{cc}
\lambda_{f} & 0 \\
0 & \lambda_{b}
\end{array}\right]=\left[\begin{array}{cc}
u+c & 0 \\
0 & u-c
\end{array}\right]
$$

where $\lambda_{f, b}$ is the forward and backward wave speed respectively. And

$$
c=\sqrt{\frac{\beta}{2 \rho}} A^{1 / 4}
$$

is the intrinsic wave speed, which is much greater than fluid velocity $u$ under physiological flow conditions and therefore the system is hyperbolic and subsonic. This needs one boundary condition each at inlet and exit of the domain. The backward and forward characteristic variables are derived as (Mynard and Nithiarasu 2008)

$$
w_{f, b}=u \pm 4 c
$$

and they are accompanied by two initial values and thus the final form is given by Low et al. (2012)

$$
w_{f, b}^{*}=w_{f, b} \pm w_{f, b}^{o}
$$

where the superscript $o$ indicates an initial value. Finally, the set of the boundary conditions is written for $A$ as

$$
A=\left[\frac{w_{f}^{*}-w_{b}^{*}}{8}+c^{o}\right]^{4}+\left(\frac{2 \rho}{\beta}\right)^{2}
$$

where $c^{o}$ is the initial state of $c$, i.e., when $A=A_{o}$ in Eq. 15, and the boundary condition for $u$ may be written as

$$
u=u^{o}+\frac{w_{f}^{*}-w_{b}^{*}}{2}
$$

Further details on boundary conditions may be obtained from references (Mynard and Nithiarasu 2008; Low et al., 2012; Coccarelli and Nithiarasu 2015;?, Coccarelli et al., 2016).

\section{Simulation Settings and Results}

In this section, some tests on the proposed semi- and fully-implicit form of LCG method are presented and compared against established explicit results. The results are divided into simple systems and full systemic circulation as discussed in the next two subsections.

\section{Simple Systems}

A simple case of wave propagation through a straight and flexible tube is considered here as shown in Fig. 2. The tube properties are $\beta=727790.92$ dyne $/ \mathrm{cm}^{3}$, $L=20 \mathrm{~cm}$ and $A_{o}=7.01 \mathrm{~cm}^{2}$. We enforce a short pressure wave (over $0.1 \mathrm{~s}$ period) with a maximum amplitude of $1000 \mathrm{dyne} / \mathrm{cm}^{2}$ as shown. The reflection coefficient at the exit is set as zero. The pressure is monitored at three points, at lengths $0.25 \mathrm{~L}, 0.5 \mathrm{~L}$ and $0: 75 \mathrm{~L}$ and they are labeled respectively as 1,2 and 3 in Fig. 2. The comparisons of the results are carried out between standard and well established LCG method with explicit time integration, implicit LCG with the interface flux calculated explicitly $\left(\mathbf{F}^{n}\right)$ and implicit LCG with interface flux also calculated implicitly $\left(\mathbf{F}^{n+1}\right)$ (i.e., respectively with $\mathbf{f}_{\Gamma}{ }^{n}=\mathbf{F}^{n}$ and $\mathbf{f}_{\Gamma}^{n+1}=\mathbf{F}^{n+1}$ as discussed in Eq. 10). The first one was developed by (Mynard and Nithiarasu 2008) and then extended by (Low et al., 2012) for more validation studies against in vivo results, while the second and third approaches are new methods as shown by Eq. 10, where the flux is estimated either at $n$ or $n+1$. The time step is adjusted to fit the requirements of stability condition of explicit method for LCG (Mynard and Nithiarasu 2008; Low et al., 2012), which was estimated to be $\Delta t=2 \times 10^{-5}$. A small time step is maintained here to compare the well established explicit method against the proposed

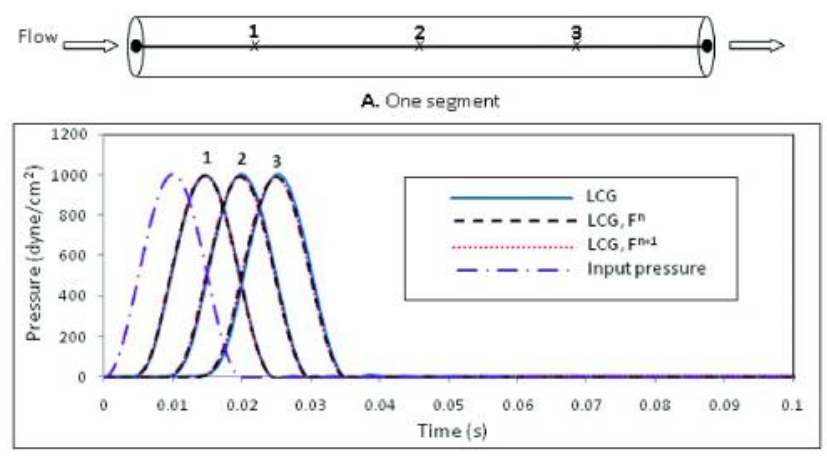

Fig. 2: Pressure wave in a single tube 
semi- and fully- implicit methods. A large time step for implicit Taylor Galerkin method will be diffusive and thus not used here. Fig. 2 shows that all methods provide almost identical results. This indicates that the new methods are as accurate as the well established explicit LCG method.

\section{Full Systemic Circulation}

The example presented in the previous subsection clearly demonstrated that the proposed new approaches are accurate for simple problems. Here, we employ the new methods on a full systemic circulation. Unless specified, all the parameters are adopted from (Mynard and Nithiarasu 2008; Low et al., 2012; Coccarelli et al., 2016). The duration of a cardiac cycle is assumed to be $0.8 s$ or $70-75$ beats/ min and the opening valve time is $0.057 \mathrm{~s}$ while the closing time is 0:039s. The input to the model is the pressure wave to the left ventricle as shown in Fig. 3.

The governing equations are solved for fluid flow in a systemic circulation model shown in Fig. 1 covering the main arteries in the body, and also coronaries and ventricular valve are included. The standard explicit time step restrictions are applied to explicit LCG method. Since the new approaches proposed are at least partially implicit, we set a slightly

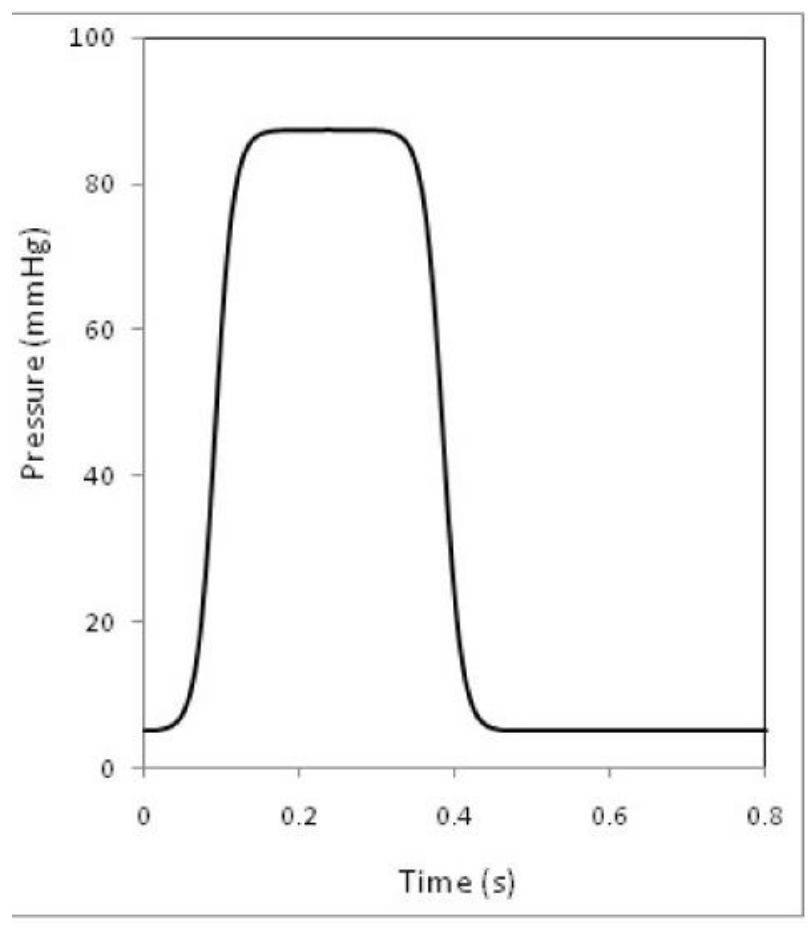

Fig. 3: Input pressure wave higher time step value of $\Delta t=0.0001 s$. Although larger time step values are possible, the time step dependent Taylor Galerkin method will dampen the solution at larger time step values.

To reinforce the validity of the proposed methods, we compare present results against the published data in (Low et al., 2012) (legend used for explicit method is LCG). The location of interest here is the mid-point of the ascending aorta (i.e. segment 9 in Fig. 1) and the pressure variation at this point is shown in Fig. 4. As seen in Fig. 4, no substantial difference between methods are noticed. This is another test that provides confidence on the new methods proposed.

For the sake of completeness, many other monitoring points of the systemic circulation are also considered here. The pressure and flow variations at different locations are shown in Figs. 5 and 6 respectively. The monitoring points considered are right and left common carotid arteries with segment numbers 13 and 23 (A and B), right and left subclavian II arteries represented by segments 15 and 29 ( $C$ and D) and left and right femoral arteries indicated by segment numbers 54 and 60 (E and F). Figure 1 shows the details of all mentioned monitoring points. As seen in Figs. 5 and 6, all methods show consistency in terms of the accuracy. Furthermore, the results demonstrate that Locally Conservative Galerkin approach is applicable in explicit, semi- and fully-implicit forms.

We also present the results of the pressure and flow for the Thoracic aorta II and abdominal aorta IV (segments 35 and 47 in Fig. 1 and respectively labelled A and B in Figs. 7 and 8) in Figs. 7 and 8 for three cardiac cycles. The time step values for the implicit LCG method is doubled to $\mathrm{Dt}=0.0002 \mathrm{~s}$ and the explicit time restriction is maintained again for explicit LCG method. Figure 7 (cases A and B) shows good agreement of pressure between all cases considered. The flow comparison in Fig. 8 also shows very good agreement between methods.

Next, we evaluate the effect of mesh refinement on the accuracy as shown in Fig. 9. The abdominal aorta $\mathrm{V}$ is chosen for this test (i.e. segment 49 in Fig. 1) and the solution is obtained using LCG, $\mathrm{F}^{\mathrm{n}+1}$ method. Clearly, the results demonstrate that all three meshes give almost identical results and and thus the solution provided here is mesh independent. 

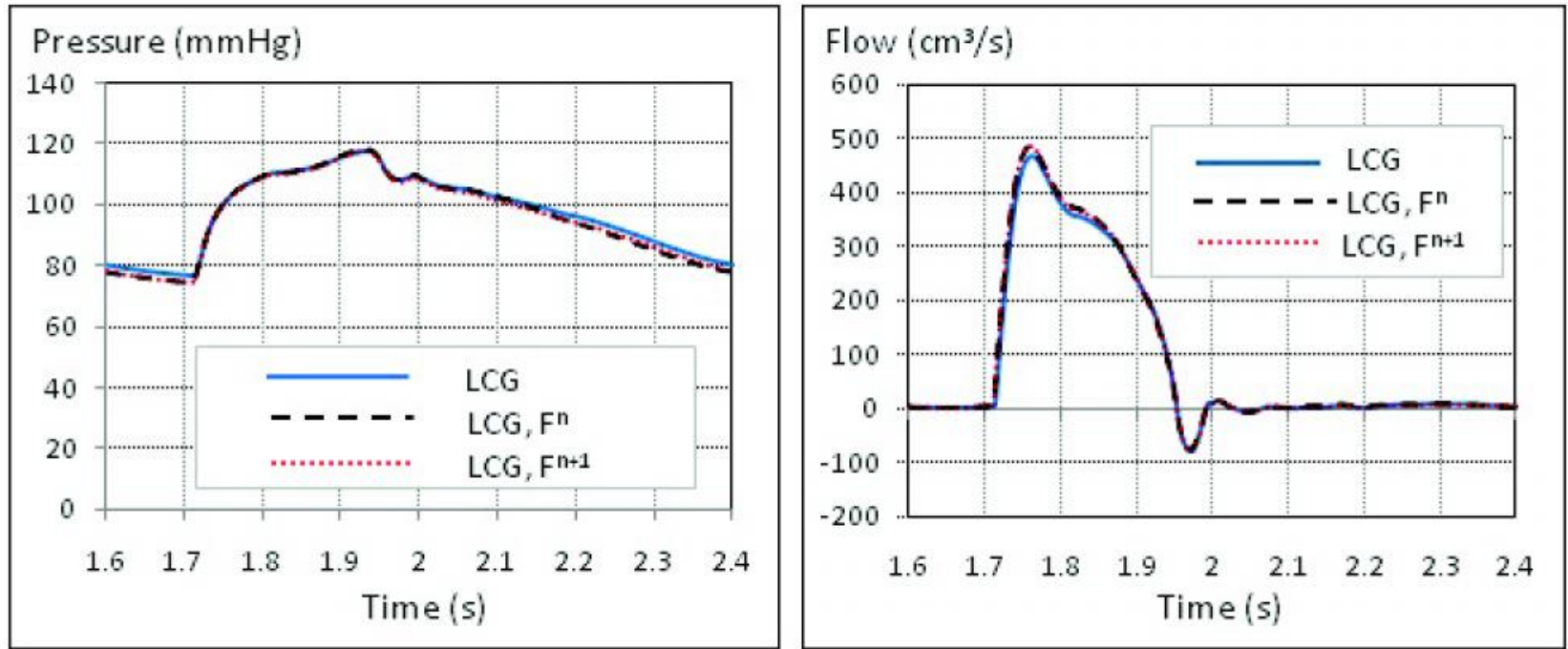

Fig. 4: Comparisons of the pressure and flow rate at the mid-point of the ascending aorta
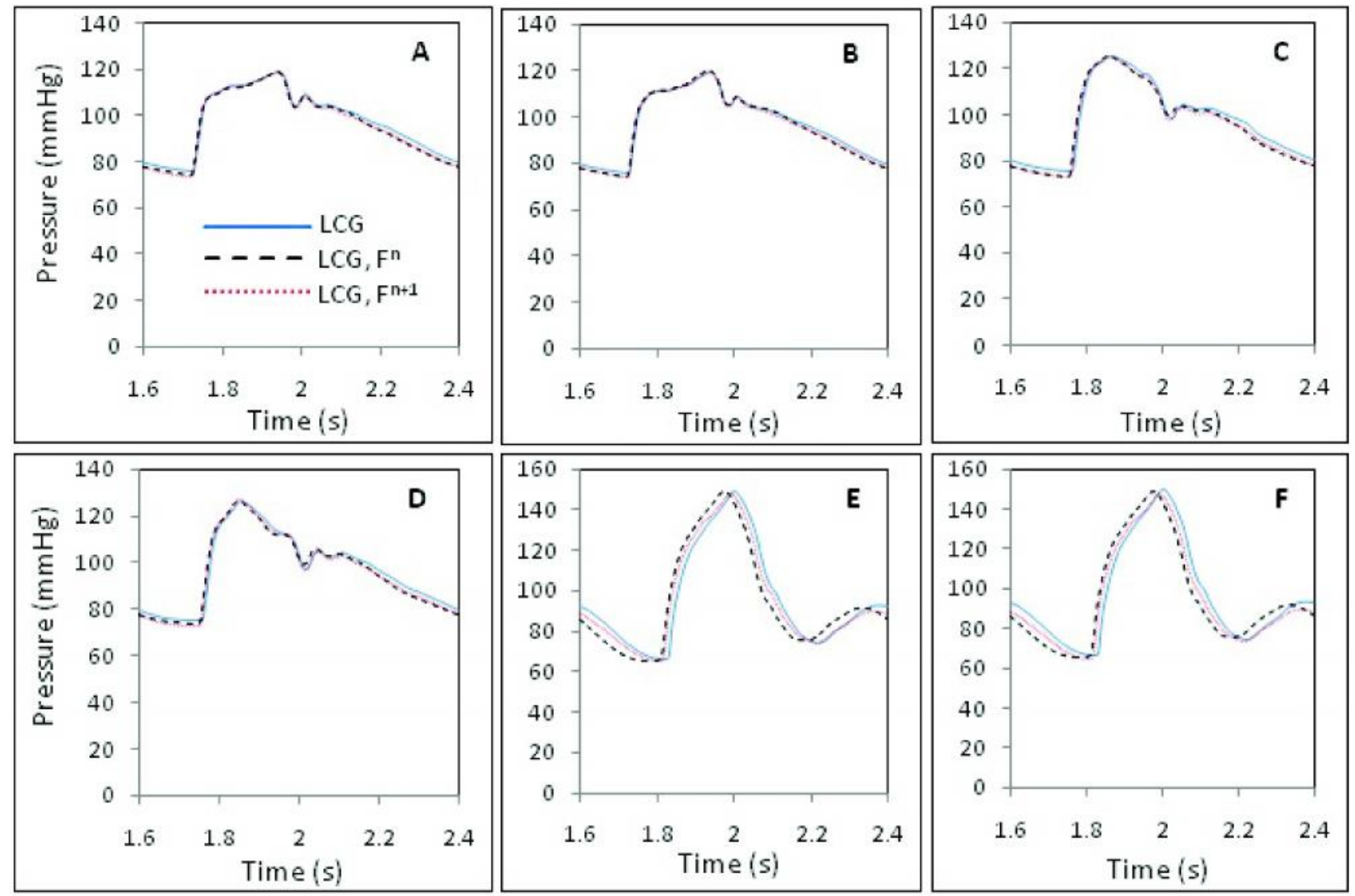

Fig. 5: Pressure variation over time at the mid-point of various arterial segments

Finally, a computational speed comparison is carried out for all methods employed and the results are shown in Table 1 . None of the previous works has revealed such comparison for explicit, semi- and full-implicit methods for a human circulatory system.
Thus, we evaluate all the presented methods for calculation speed on a mesh with 6288 elements. The results clearly shows that both the new methods (i.e. LCG, $\mathrm{F}^{\mathrm{n}}$ and $\mathrm{LCG}, \mathrm{F}^{\mathrm{n}+1}$ ) are faster and can admit much higher time steps than that of the explicit LCG method. 

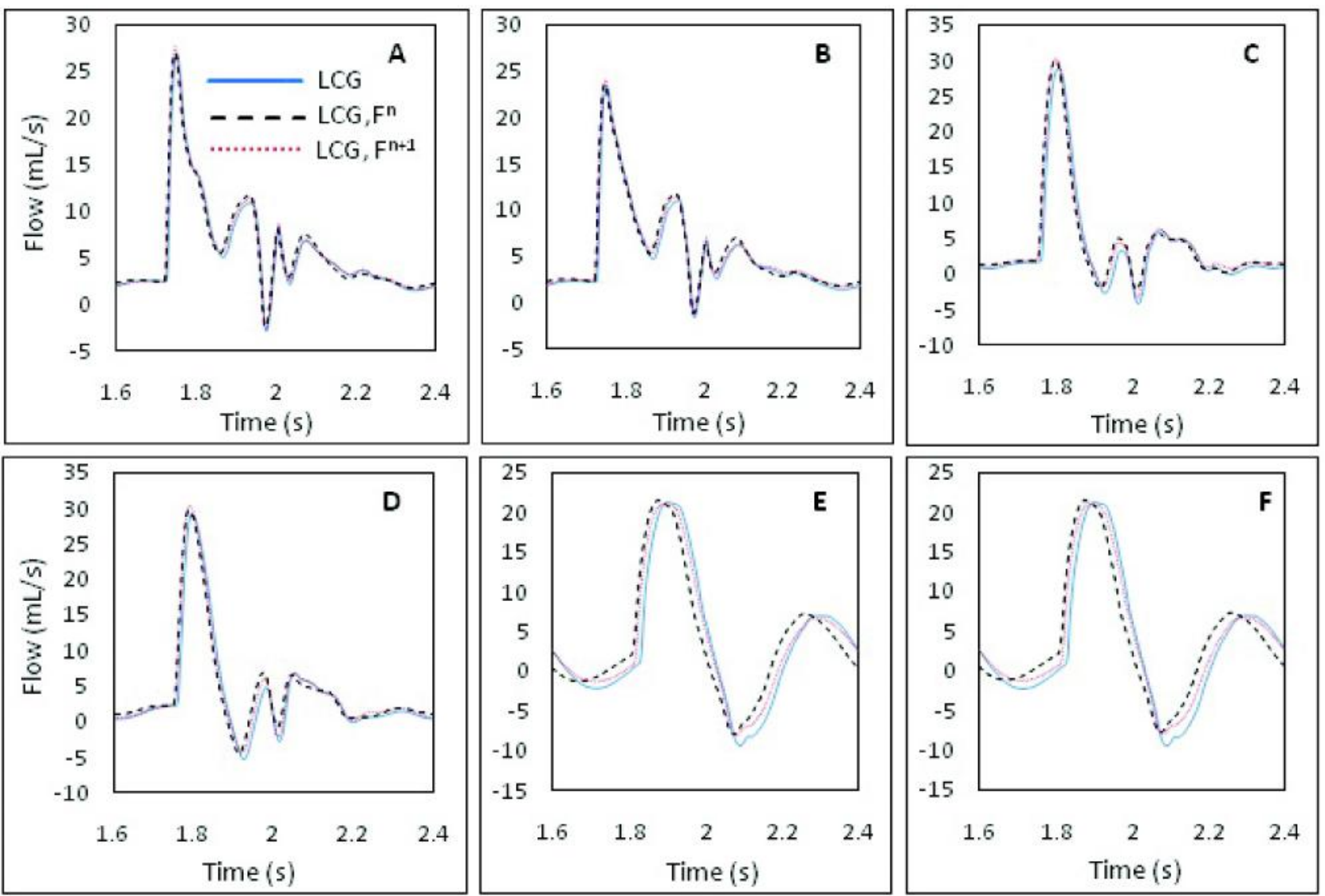

Fig. 6: Flow rate variation over time in the middle of various arterial segments


Fig. 7: Pressure variation over time in the middle of various arterial segments

Fig. 8: Flow rate variation over time in the middle of various arterial segments

Due to the fact that the boundary conditions employed here are based on extrapolation of characteristic variables from previous time step, the standard method are very difficult to implement. However, the implicit

LCG method appears to be easy to implement and provides accurate solution. This may be due to the fact that the matrix size never exceeds the element matrix size and simplifies calculations. 


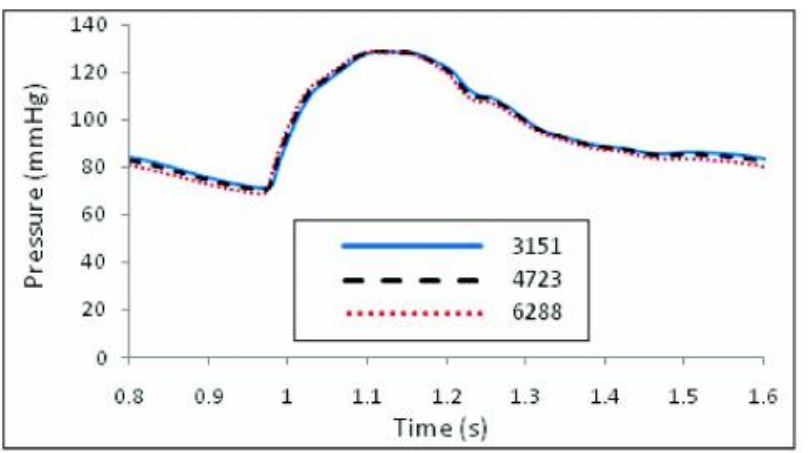

Fig. 9: Pressure variation over time in the middle of Abdominal Aorta V

Table 1: Computational speed comparison averaged over three cardiac cycles

\begin{tabular}{lccc}
\hline Method & LCG, $F^{n}$ & LCG, $F^{n+1}$ & LCG \\
\hline Time (s) & 918.75 & 873.75 & 1017.00 \\
\hline
\end{tabular}

\section{References}

Alastruey J, Parker K H, Peiro J, Byrd S M and Sherwin S J (2007) Modelling the circle of willis to assess the effects of anatomical variations and occlusions on cerebral flows Journal of Biomechanics 40 1794-1805

Avolio A (1980) Multi-branched model of the human arterial system Medical and Biological Engineering and Computing 18 709-718

Blanco P J, Leiva J S, Feijo R A and Buscaglia G C (2011) Blackbox decomposing approach for computational hemodynamic: One-dimensional model Computer Methods in Applied Mechanics and Engineering 200 1389-1405

Blanco P J, Trenhago P R, Fernandes L G and Feijo R A (2012) On the integration of the baroreflex control mechanism in a heterogeneous model of the cardiovascular system, International Journal for Numerical Methods in Biomedical Engineering 28 412-433

Boileau E, Nithiarasu P, Blanco J, Muller L, Fossans F E, Helleviks L, Doners W, Huberts W, Willemet M and Alastruey J (2015) A benchmark study of 1-d numerical schemes for arterial blood flow modelling International Journal for Numerical Methods in Biomedical Engineering e02732(DOI: 10.1002/cnm.2732)

Chen P, Quarteroni A and Rozza G (2013) Simulation-based uncertainty quantification of human arterial network hemodynamics International Journal for Numerical

\section{Concluding Remarks}

The explicit, semi- and fully-implicit LCG method with the Taylor-Galerkin stabilization have been discussed for systematic circulation studies. The semi- and fullyimplicit solutions have been achieved by adopting Newton-LU iteration for flow. Such a technique, along with LCG spatial discretisation produces very small matrix, which allows us to use any standard version of Newton method with very fast convergence. The proposed new methods are faster than standard explicit LCG method and are accurate. Further tests on the new methods are needed to reduce the difficulties associated with higher time steps (damping).

\section{Acknowledgement}

The authors acknowledge the financial support provided by the Iraqi Government and partial support provided by the Ser Cymru National Research Network in Advanced Engineering and Materials.

Methods in Biomedical Engineering 29 698-721

Coccarelli A, Boileau E, Parthimos D and Nithiarasu P (2016) An advanced computational bioheat transfer model for a human body with an embedded systemic circulation Biomechanics and Modeling in Mechanobiology (to appear) (DOI: 10.1007/s10237-015-0751-4)

Coccarelli A and Nithiarasu P (2015) A robust finite element modelling approach to conjugate heat transfer in flexible elastic tubes and tube networks Numerical Heat Transfer, Part A Applications 67 513-530

Formaggia L, Nobile F and Quarteroni A(2002) A one dimensional model for blood flow: Application to vascular prosthesis, in I. Babuska T., Miyoshi and P. Ciarlet eds, Mathematical modeling and numerical simulation in continuum mechanics, lecture notes in computational science and engineering Springer-Verlag, Berlin

Franke V, Peiro J, Sherwin S, Ling W and Fisk N (2002) Computational modelling of 1D blood flow and its applications ESIAM Proceedings, M Thiriet 12 48-54

Kufahl R and Clark M (1985) A circle of willis simulation using distensible vessels and pulsatile flow Journal of Biomechanical Engineering 107 112-122

Low K, van Loon R, Sazonov I, Bevan R L T and Nithiarasu P (2012) An improved baseline model for a human arterial network to study the impact of aneurysms on pressureflow waveforms International Journal for Numerical 
Methods in Biomedical Engineering 28 1224-1246

Malossi A C I, Blanco P J and Deparis S (2012) A two-level time step technique for the partitioned solution of onedimensional arterial networks Computer Methods in Applied Mechanics and Engineering 237-240 212-226

Mynard J and Nithiarasu P (2008) A 1D arterial blood flow model incorporating ventricular pressure, aortic valve and regional coronary flow using locally conservative Galerkin (LCG) method Communications in Numerical Methods in Engineering 24 367-417

Nithiarasu P (2004) A simple locally conservative galerkin (LCG) finite-element method for transient conservation equations Numerical Heat Transfer Part B - Fundamentals 46357 370

Nithiarasu P, Lewis R and Seetharamu K (2016) Fundamentas of the finite element method for heat, mass and fluid flow, second edn, Wiley

Olufsen M, Peskin C, Kim W, Pedersen E, Nadim A and Larsen J (2000) Numerical simulation and experimental validation of blood flow in arteries with structured-tree outflow conditions Annals of Biomedical Engineering 28 12811299

Raines J, Jaffrin M and Shapiro A (1974) A computer simulation of arterial dynamics in the human leg Journal of Biomechanics 7 77-91

Sherwin S, Formaggia L, Peiro J and Franke V (2003) Computational modelling of $1 \mathrm{D}$ blood flow with variable mechanical properties and its application to the simulation of wave propagation in the human arterial system International Journal for Numerical Methods in Fluids $\mathbf{4 3}$ 673-700

Steele B N, Olufsen M S and Taylor C A (2007) Fractal network model for simulating abdominal and lower extremity blood flow during resting and exercise conditions Computer Methods in Biomechanics and Biomedical Engineering 10 39-51

Stergiopulos N, Young D and Rogge T (1992) Computer simulation of arterial flow with applications to arterial and aortic stenoses Journal of Biomechanics 25 1477-1488

Thomas C G, Nithiarasu P and Bevan R L T (2008) The locally conservative galerkin (lcg) method for solving the incompressible navier-stokes equations International Journal for Numerical Methods in Fluids 57 1771-1792

Urquiza S A, Blanco P J, Venere M J and Feijoo R A (2006) Multidimensional modelling for the carotid artery blood flow Computer Methods in Applied Mechanics and Engineering 195 4002-4017

Wan J, Steele B, Spicer S, Strohband S, Feijoo G, Hughes T and Taylor C (2002) A one dimensional finite element method for simulation-based medical planning for cardiovascular disease Computer Methods in Biomechanics and Biomedical Engineering 5 195-206

Wanga X, Fullanaa J and Lagree P (2015) Verification and comparison of four numerical schemes for a $1 \mathrm{~d}$ viscoelastic blood flow model Computer Methods in Biomechanics and Biomedical Engineering 18 1704-1725

Watanabe S M, Blanco P J and Feijo R A (2013) Mathematical module of blood flow in an anatomically detailed network of the arm M2AN 47 961-985

Zienkiewicz O C, Taylor R L and Nithiarasu P (2014) The finite element method for fluid dynamics, seventh edn, Elsevier

Zienkiewicz O, Taylor R and Fox D (2014) The finite element method for solid and structural mechanics, seventh edn, Elsevier. 